\title{
ARTIGO ORIGINAL Sintomatologia depressiva no termo da gestação, em mulheres de baixo risco
}

\author{
Depressive symptomatology at full-term pregnancy in low risk women \\ Vanessa Silva ${ }^{1,2}$ \\ https://orcid.org/0000-0001-8322-3952 \\ Cátia Ferreira' \\ https://orcid.org/0000-0003-0201-6033 \\ Adriana Basílio ${ }^{3}$ \\ Ana Beatriz Ferreira ${ }^{3}$ \\ Berta Maia ${ }^{4}$ \\ https://orcid.org/0000-0002-6640-6033 \\ Rui Miguelotet,2 \\ https://orcid.org/0000-0001-7710-5677
}

\section{RESUMO}

Objetivos: Calcular a prevalência de sintomatologia depressiva pré-natal em grávidas de baixo risco, no termo da gestação, avaliar seus preditores e desfechos materno-fetais. Para tal, avaliar-se-á a aplicabilidade da Escala de Rastreio de Depressão Pós-Parto (PDSS 24) nessa fase da gravidez. Métodos: A PDSS 24 e um questionário sociodemográfico, psicossocial e médico (antecedentes obstétricos e patológicos) foram autoaplicados a 403 grávidas (37-40 semanas de gestação), com idade média de 30,5 anos (DP =4,67). Por meio do processo clíniCo, foram recolhidos dados de resultados materno-fetais. Resultados: A PDSS 24 possui propriedades psicométricas adequadas para a deteção de sintomatologia depressiva pré-natal. A prevalência de sintomatologia depressiva pré-natal foi de 41,7\%. Grávidas com níveis de escolaridade inferiores, não casadas, cuja gravidez não foi planejada e com antecedentes de acontecimentos de vida significativos apresentam risco duas vezes superior de sintomatologia depressiva no período pré-natal. Grávidas cujo apoio social percebido ao longo da gravidez não correspondeu ao desejado e com história prévia de depressão apresentam cerca de três vezes

\section{Palavras-chave:}

Depressão pré-natal, sintomatologia depressiva, rastreio, Escala de Rastreio de Depressão Pós-Parto, prevalência, fatores associados. maior risco sintomatologia depressiva no período pré-natal. Para desfechos materno-fetais (pré-eclâmpsia, restrição de crescimento fetal, Apgar 1\%/50 minuto, tipo de parto, percentil de peso, oligoâminos e necessidade de cuidados intensivos), as diferenças foram não significativas. Conclusão: $O$ rastreio da depressão pré-natal deve ser realizado na gravidez. Porém, no termo da gestação o uso da PDSS 24 como ferramenta de deteção de sintomatologia depressiva deve ser feito com cautela. A elevada prevalência de sintomas relacionados com o sono nessa fase da gestação pode conduzir ao sobre diagnóstico, usando a PDSS 24.

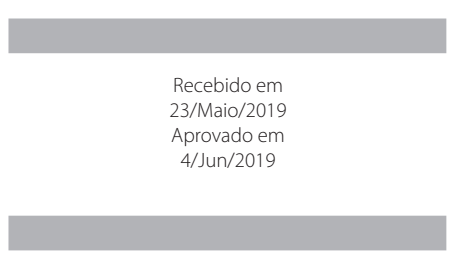

DOI: $10.1590 / 0047-2085000000229$
1 Hospital Senhora da Oliveira - Guimarães, Creixomil, Portugal.

2 Escola de Medicina da Universidade do Minho - Instituto de Ciências da Vida e da Saúde (ICVS), Braga, Portugal.

3 Escola de Medicina da Universidade do Minho, Braga, Portugal.

4 Universidade Católica Portuguesa, Faculdade de Filosofia e Ciências Sociais, Centro Regional de Braga, Braga, Portugal. 


\section{Keywords:}

Prenatal depression, depressive symptomatology, screening, Postpartum Depression Screening Scale, risk factors, adverse outcomes.

\section{ABSTRACT}

Objectives: The aims of the study were to estimate the prevalence of depressive symptomatology in full-term pregnancy (low risk), evaluate their predictors and maternal-fetal outcomes. To this end, the applicability of Postpartum Depression Screening Scale (PDSS 24) will be evaluated, at full-term pregnancy. Methods: PDSS 24 and a sociodemographic, psychosocial, pathological and obstetrical background questionnaire were self-administered to 403 pregnant women (37-40 weeks gestation), with a mean age of 30.5 years ( $S D=4.67)$. Data from maternal, fetal and neonatal outcomes were collected from the patient clinical process. Results: PDSS 24 revealed adequate psychometric properties to screening depressive symptomatology in full-term pregnancy. The prevalence of depressive symptomatology was $41.7 \%$. Pregnant women with lower study levels, who weren't married, whose pregnancy was unplanned and with a previous history of significant life events present twice the risk to present depressive symptomatology. Pregnant women who hadn't received the desired social support in pregnancy and with a history of depression present about a 3-fold increased risk to present depressive symptomatology. For maternal-fetal outcomes (pre-eclampsia, fetal growth restriction, Apgar score at $1^{\text {st }} / 5^{\text {th }}$ minute, type of delivery, weight percentile, oligohydramnios and need for neonatal intensive care), the differences were not significant. Conclusion: Screening for prenatal depression should be conducted during pregnancy. However, in full-term pregnancy women, the use of PDSS 24 as a screening tool for depressive symptomatology should be done with caution. The high prevalence of sleep-related symptoms, in full-term pregnancy, may lead to overdiagnosis, using PDSS 24.

\section{INTRODUÇÃO}

A depressão perinatal é atualmente reconhecida como um problema de saúde pública', que pode ocorrer a qualquer momento entre a concepção até ao primeiro ano após o parto.

A investigação científica centrou-se preferencialmente na análise da depressão pós-parto, tornando a depressão durante a gravidez uma questão secundária. No entanto, são vários os motivos apontados na literatura atual para o estudo da depressão pré-natal:

- Primeiro: elevada prevalência. Estima-se que 25\% das grávidas apresentem sintomatologia depressiva, a prevalência de depressão pré-natal ronde os 8,5\% a 11\%, e 3,1\% a 4,9\% das grávidas sofram de depressão major;

- Segundo: falha no diagnóstico e tratamento. Aproximadamente $75 \%$ dos casos de depressão pré-natal não são diagnosticados ${ }^{3}$. Os distúrbios de sono, alterações de apetite, do nível de energia e de interesse presentes na depressão pré-natal são muitas vezes confundidos como alterações normais da gravidez, quer pela grávida, quer pelos profissionais de saúde 4 . Por outro lado, sem programas de rastreio apenas 10\% das grávidas são tratadas5;

- Terceiro: estigma social e falta de divulgação da patologia. Apenas uma em cada cinco grávidas com depressão procura ajuda médica ${ }^{6}$;

- Quarto: depressão pré-natal é um fator consistente para depressão pós-parto 78 , tendo a depressão perinatal especificidades comparativamente à depressão noutros períodos de vida ${ }^{4,9,10}$;
- Quinto: potenciais consequências negativas da depressão pré-natal, com repercussões na saúde materna (pré-eclâmpsia, complicações no parto) ${ }^{11,12}$, fetal (baixo peso, prematuridade) ${ }^{13,14}$, bem como na dinâmica familiar ${ }^{15}$.

Com o reconhecimento do continuum entre depressão durante a gravidez e no período pós-parto e as suas consequências pré e pós-natais, torna-se vital o rastreio de sintomatologia depressiva pré-natal. Com essa finalidade, é utilizada a Escala de Rastreio de Depressão Pós-Parto (PDSS) ${ }^{16}$, que, embora não tenha sido desenvolvida para a gravidez, é considerada um instrumento válido para o rastreio de sintomatologia depressiva pré-natal17. Essa escala mostra-se particularmente útil na identificação de grávidas que se beneficiam de apoio especializado, bem como na avaliação da sobreposição entre sintomatologia da gravidez e sintomatologia depressiva ${ }^{17}$.

O aumento do contato entre grávida-profissional de saúde durante a gravidez aumenta as oportunidades para o rastreio da sintomatologia depressiva pré-natal18 e referenciação para avaliação diagnóstica de depressão pré-natal. O correto diagnóstico e a intervenção precoce levam à redução dos sintomas depressivos nesse período ${ }^{19}$, com redução dos custos a longo prazo, resultantes das consequências negativas da depressão perinatal ${ }^{20}$.

Apesar da crescente evidência científica nessa área, há falta de estudos da prevalência de sintomatologia depressiva período pré-natal, em especial no termo da gestação, bem como dos seus fatores associados, na população portuguesa 
de grávidas de baixo risco. É fundamental a sensibilização e início do rastreio da sintomatologia depressiva no período pré-natal, como primeiro passo para a deteção de depressão perinatal.

Pretendemos com este estudo responder às seguintes questões:

1. Será a PDSS 24 adequada para o rastreio de sintomatologia depressiva no termo da gestação, em grávidas de baixo risco?

2. Qual a prevalência de sintomatologia depressiva no período pré-natal, a termo da gestação, em grávidas de baixo risco?

3. Existe associação entre sintomatologia depressiva e fatores sociodemográficos, psicossociais e obstétricos?

4. Existe associação entre sintomatologia depressiva e os desfechos materno-fetais?

\section{MÉTODOS}

\section{Amostra}

A amostra é constituída por 403 mulheres grávidas de baixo risco, no terno da gestação (37 a 41 semanas), avaliadas na consulta de gestação de termo do Hospital Senhora da Oliveira - Guimarães. A média de idades maternas é de 30,5 anos $(\mathrm{DP}=4,67)$, sendo a maioria das grávidas casada $(78,9 \%)$ e vivendo com o companheiro (71\%). 31,5\% concluíram o $12^{\circ}$ ano de escolaridade e 78,7\% encontram-se empregadas (Tabela 1).

\section{Procedimento}

Definiram-se como critérios de inclusão: idade mínima de 18 anos, inclusive, e idade gestacional entre 37 e 41 semanas. Excluíram-se grávidas com qualquer condição médica que exija ou exigiu vigilância em consulta hospitalar da especialidade de obstetrícia e o não entendimento de língua portuguesa.

Todas as grávidas que preencheram os critérios enunciados foram convidadas a colaborar neste estudo, assinando o Consentimento Informado. A administração do questionário (constituído por duas partes) decorreu no período de espera entre a avaliação de enfermagem e a avaliação médica, entre os meses de abril e dezembro de 2016. As duas partes do questionário foram autoaplicadas, o tempo médio de resposta foi de 15 minutos. O anonimato foi sempre garantido. O protocolo de estudo foi aprovado pela Comissão de Ética do Hospital Senhora da Oliveira - Guimarães.

\section{Instrumentos}

A presença de sintomatologia depressiva pré-natal foi avaliada por meio da Escala de Rastreio de Depressão Pós-Parto $(P D S S)^{16}$ (primeira parte do questionário), desenvolvida por Beck e Gable em 2002, traduzida e validada para a versão
Tabela 1. Análise descritiva das características sociodemográficas, psicossociais e obstétricas da amostra $(n=403)$

\begin{tabular}{|c|c|}
\hline Variáveis & Análise descritiva \\
\hline Idade materna ${ }^{a}$ & $18-41(30,4 \pm 4,67)$ \\
\hline \multicolumn{2}{|l|}{ Escolaridade $^{b}$} \\
\hline $1^{\circ}$ ao $4^{\circ}$ ano & $8(2)$ \\
\hline $5^{\circ}$ ao $6^{\circ}$ ano & $102(25,3)$ \\
\hline $7^{0}$ ao $9^{\circ}$ ano & $116(28,8)$ \\
\hline $10^{\circ}$ ao $12^{\circ}$ ano & $127(31,5)$ \\
\hline Ensino universitário & $50(12,4)$ \\
\hline \multicolumn{2}{|l|}{ Empregabilidade $^{b}$} \\
\hline Desempregada & $85(21,1)$ \\
\hline \multicolumn{2}{|l|}{ Estado civil $^{b}$} \\
\hline Solteira & $65(16,1)$ \\
\hline Casada/união de fato & $318(78,9)$ \\
\hline Divorciada & $15(3,7)$ \\
\hline Viúva & $3(0,7)$ \\
\hline \multicolumn{2}{|l|}{ Apoio social percebido ${ }^{b}$} \\
\hline Não & $41(10,2)$ \\
\hline \multicolumn{2}{|c|}{ Acontecimentos de vida prévios traumáticos ${ }^{b}$} \\
\hline $\operatorname{Sim}$ & $65(16,1)$ \\
\hline \multicolumn{2}{|c|}{ Antecedentes de depressã $0^{b}$} \\
\hline $\operatorname{Sim}$ & $58(14,5)$ \\
\hline Idade gestacional ${ }^{\mathrm{a}}$ & $37-40(37,9 \pm 0,71)$ \\
\hline \multicolumn{2}{|l|}{ Paridade $^{b}$} \\
\hline Nulípara & $238(59,1)$ \\
\hline \multicolumn{2}{|c|}{ Planejamento da gravidez atual $^{b}$} \\
\hline Não & $69(17,2)$ \\
\hline \multicolumn{2}{|l|}{ História de abortamento $0^{\mathrm{b}}$} \\
\hline Sim & $48(11,9)$ \\
\hline \multicolumn{2}{|c|}{ Complicações obstétricas anteriores ${ }^{b}$} \\
\hline $\operatorname{Sim}$ & $32(7,9)$ \\
\hline
\end{tabular}

bFrequência absoluta e frequência relativa (\%)

portuguesa por Pereira e colaboradores em $2009^{17}$. Esse instrumento de autoaplicação, composto por 35 itens avaliados numa escala de Likert, foi desenvolvido para a avaliação da presença de sintomatologia depressiva no pós-parto, indicando a necessidade de avaliação diagnóstica adicional. Quanto mais elevada a pontuação (variando entre 35 e 175 pontos), maior a gravidade da sintomatologia; pontuações baixas sugerem poucos ou nenhum sintoma depressivo, logo, um ajustamento normal ao período do pós-parto. Quanto à utilização da PDSS na gravidez, a escala mostra boas caraterísticas psicométricas e excelentes caraterísticas operatórias, mostrando ser adequada para o rastreio de sintomatologia depressiva pré-nata ${ }^{17}$. O mesmo acontece com a sua versão curta, de 24 itens, validada por Pereira e colabo- 
radores em $2013^{21}$, tendo sido esta a versão utilizada neste estudo (PDSS 24). Para o rastreio de sintomatologia depressiva pré-natal (de acordo com o CID-10 e ajustada para a prevalência na população) ${ }^{21}$, foi considerado como ponto de corte o valor 43, por apresentar maior especificidade (79,2\%), sensibilidade $(85,2 \%)$, valor preditivo positivo $(11,9 \%)$ e negativo (99,4\%), menor número de falso-positivos $(20,8 \%)$ e falso-negativos $(14,3 \%)^{21}$.

A segunda parte do questionário de autoaplicação, relativo a fatores psicossociais e antecedentes patológicos e obstétricos, foi construída com base em pesquisas sobre a literatura da especialidade. Recolheram-se ainda dados sociodemográficos. Com o intuito de avaliar a influência da sintomatologia depressiva pré-natal nos resultados obstétricos e materno-fetais, mais preponderantes segundo a literatura, foram recolhidos dados por meio do processo clínico electrônico da grávida, contidos nos programas informáticos SClínico e Obscare .

\section{Tipo de estudo e análise estatística}

Trata-se de um estudo não experimental, transversal, quantitativo, descritivo e correlacional, com uma amostra não probabilística de conveniência.

A análise estatística foi realizada por meio do programa SPSS $^{\circ}$, versão 25.0. O coeficiente de alfa de Cronbach foi usado para avaliar a consistência interna da PDSS 24 e das suas dimensões (F1 - Falha e desrealização, F2 - Dificuldades de concentração e ansiedade, F3 - Ideações suicidas, F4 - Dificuldades em dormir), considerando-se valores entre 0,65 e 0,70 como aceitáveis, 0,70 e 0,80, bons, 0,80 e 0,90, muito bons, e valores maiores que 0,90 mostram elevada confiabilidade ${ }^{17}$. Para a descrição demográfica e o cálculo da prevalência de sintomatologia depressiva pré-natal (PDSS $\geq 43$ ), realizou-se uma análise descritiva. Como análise descritiva univarida, foram determinados média (M) e desvio-padrão (DP) para variáveis quantitativas e frequências absolutas (n) e relativas (\%) para variáveis qualitativas. Na análise bivariada, para variáveis quantitativas, foi utilizado o teste $t$-student (t) para amostras independentes e o teste de qui-quadrado (X2) para variáveis qualitativas. Para avaliar possíveis preditores de sintomatologia depressiva pré-natal e a sua influência nos resultados obstétricos e materno-fetais, realizou-se uma regressão logística binária. Para todas as análises, estabeleceu-se um nível de significância estatística ( $p$-value) inferior a 0,05 e um intervalo de confiança (IC) de 95\%.

\section{RESULTADOS}

\section{Propriedades psicométricas da PDSS 24 no termo da gestação}

Neste estudo, a PDSS 24 revelou elevada consistência interna, com um alfa de Cronbach de 0,913, com todos os itens varian- do entre os valores de 0,907 e 0,918. Quanto às questões "tive dificuldade em dormir" e "acordei a meio da noite e tive dificuldade em voltar a adormecer", a sua remoção levaria à melhoria da consistência interna, avaliada pelo alfa de Cronbach, que aumentaria para 0,915 e 0,918, respetivamente. Avaliando a PDSS 24 e as suas dimensões, verificou-se boa consistência interna global, com um alfa de Cronbach de 0,769, com todos F's variando entre 0,691 (F2) e 0,828 (F4). Caso F4 fosse removido, a consistência interna aumentaria de 0,769 para 0,828.

\section{Prevalência de sintomatologia depressiva no termo da gestação}

Das 403 participantes em estudo, 168 manifestaram sintomatologia depressiva (PDSS $24 \geq 43$ ), o que corresponde a $41,7 \%$ da amostra.

\section{Características sociodemográficas, obstétricas e psicossociais}

A maioria das grávidas considera que recebeu o apoio esperado durante a gestação (89,8\%). 59,1\% das grávidas são nulíparas, sendo a idade gestacional média, aquando da avaliação de 37,9 semanas ( $D P=0,71)$. 17,2\% referem que a gestação atual não foi planejada. Uma minoria de grávidas apresenta antecedentes obstétricos de relevo, nomeadamente abortamento $(11,2 \%)$ ou complicações obstétricas nas gestações anteriores (7,9\%). Por outro lado, 14,5\% apresentam antecedentes de patologia psiquiátrica, especificamente depressão, não tendo nenhuma das grávidas realizado terapêutica antidepressiva na gestação atual (Tabela 1).

\section{Associação entre sintomatologia depressiva e variáveis sociodemográficas, psicossociais e obstétricas}

Foram encontradas diferenças estatisticamente significativas entre grávidas com sintomatologia depressiva e as variáveis estado civil, escolaridade, apoio social percebido, acontecimentos de vida significativos prévios, planejamento da gravidez e história prévia de depressão, como pode ser observado na tabela 2.

\section{Preditores de sintomatologia depressiva no termo da gestação}

A regressão logística binária foi realizada para as variáveis de modo a obter um modelo de regressão mais parcimonioso. O estado civil - solteira $(p=0,044)$, escolaridade inferior ao nono ano de escolaridade $(p=0,026)$, considerar não ter recebido o apoio social esperado durante a gravidez ( $p=$ $0,026)$, gravidez não planejada $(p=0,044)$ e história prévia de depressão $(p=0,025)$ revelaram ser preditores independentes de sintomatologia depressiva no termo da gestação. Analisando a tabela 3, observa-se que o antecedente de depressão e o apoio social percebido são os preditores com maior contribuição preditiva. 
Tabela 2. Associação entre sintomatologia depressiva e fatores sociodemográficos, psicossociais e obstétricos

\begin{tabular}{|c|c|c|c|c|}
\hline Variáveis ${ }^{\mathrm{a}, \mathrm{b}}$ & $\mathrm{n}$ & $\begin{array}{l}\text { PDSS } 24 \\
\geq 43\end{array}$ & $\begin{array}{c}\text { PDSS } 24 \\
<43\end{array}$ & $\begin{array}{c}\text { Estatística' } \\
\text { (p) }\end{array}$ \\
\hline \multicolumn{5}{|l|}{ Estado civil } \\
\hline Solteira/viúva/divorciada & 401 & $46(11,5)$ & $37(9,2)$ & 0,005 \\
\hline Casada/união de fato & & $122(30,4)$ & $196(48,9)$ & \\
\hline \multicolumn{5}{|l|}{ Escolaridade } \\
\hline $1^{\circ}$ ao $9^{\circ}$ ano & 403 & $58(14,4)$ & $52(12,9)$ & 0,006 \\
\hline $10^{\circ}$ ano ao ensino universitário & & $110(27,3)$ & $183(45,4)$ & \\
\hline \multicolumn{5}{|l|}{ Apoio social percebido } \\
\hline $\operatorname{Sim}$ & 402 & $141(35,1)$ & $220(54,7)$ & 0,003 \\
\hline Não & & $26(6,5)$ & $15(3,7)$ & \\
\hline \multicolumn{5}{|c|}{ Acontecimentos de vida prévios traumáticos } \\
\hline Sim & 403 & $36(8,9)$ & $29(7,2)$ & 0,014 \\
\hline Não & & $132(32,8)$ & $206(51,1)$ & \\
\hline \multicolumn{5}{|l|}{ Planejamento da gravidez } \\
\hline Sim & 401 & $128(31,9)$ & $204(50,9)$ & 0,011 \\
\hline Não & & $38(9,5)$ & $31(7,7)$ & \\
\hline \multicolumn{5}{|l|}{ Antecedentes de depressão } \\
\hline $\operatorname{Sim}$ & 403 & $33(8,2)$ & $132(32,8)$ & 0,002 \\
\hline Não & & $25(6,2)$ & $(2,8)$ & \\
\hline
\end{tabular}

Frequência absoluta e frequência relativa (\%).

Optou-se por representar na tabela apenas as associações estatisticamente significativas. $P<0,05$,

'Teste de Qui-Quadrado.

$p=$ nível de significância

Tabela 3. Modelo de regressão logística binária para avaliar preditores de sintomatologia depressiva no termo da gestação

\begin{tabular}{lccccc}
\hline Variáveis & B & Wald & $p$ & $\operatorname{Exp}(\mathbf{B})$ & aORa $^{\text {(IC } 95 \%) ~}$ \\
\hline Estado civil & 0,532 & 4,057 & 0,044 & 1,702 & 0,702 \\
& & & & & $(1,014 ; 2,854)$ \\
Escolaridade & 0,527 & 4,969 & 0,026 & 1,694 & $\begin{array}{c}0,699 \\
(1,066 ; 2,691)\end{array}$ \\
& & & & & 0,459 \\
Gravidez planejada & 0,255 & 2,259 & 0,044 & 1,729 & $(1,276 ; 2,12)$ \\
& & & & & 0,658 \\
Acontecimentos de vida & 0,506 & 3,082 & 0,079 & 1,658 & $(0,943 ; 2,915)$ \\
significativos & & & & & 1,215 \\
Apoio social percebido & 0,795 & 4,927 & 0,026 & 3,115 & $(1,098 ; 4,472)$ \\
& & & & & 2,587 \\
Antecedentes de depressão & 0,857 & 4,568 & 0,025 & 3,388 & $(1,698 ; 2,548)$ \\
& & & & &
\end{tabular}

Constante: $B=-0,837 ; R^{2}=0,061 ; 0,083$ (Nagelkerke); $X^{2}=25,226, p<0,01$.

Ajustado para a idade materna.

p: nível de significância; aOR: odds ratio ajustado; |C: intervalo de confiança.

\section{Associação entre sintomatologia depressiva e desfechos materno-fetais}

Para os desfechos materno-fetais (pré-eclâmpsia, oligoâminos, restrição de crescimento fetal, percentil de peso, tipo de parto, Apgar ao 10 e $5^{\circ}$ minuto inferior a 7 e necessidade de cuidados intensivos neonatais), não foram encontradas diferenças significativas, como consta da tabela 4.
Tabela 4. Associação entre sintomatologia depressiva e os desfechos materno-fetais

\begin{tabular}{|c|c|c|c|c|}
\hline Variáveis & $\mathrm{n}$ & PDSS $24 \geq 43$ & PDSS $24<43$ & Estatística ${ }^{b, c}(p)$ \\
\hline \multicolumn{5}{|c|}{ Pré-eclâmpsia ${ }^{a}$} \\
\hline $\operatorname{Sim}$ & \multirow{2}{*}{403} & $3(0,7)$ & $3(0,7 \%)$ & \multirow{2}{*}{0,677} \\
\hline Não & & $165(40,9)$ & $232(57,6)$ & \\
\hline \multicolumn{5}{|c|}{ Oligoâminos ${ }^{\mathrm{a}}$} \\
\hline $\operatorname{sim}$ & 403 & $2(0,5)$ & $2(0,5)$ & \multirow[t]{2}{*}{0,735} \\
\hline Não & & $166(41,2)$ & $233(57,8)$ & \\
\hline \multicolumn{5}{|c|}{ Restrição de crescimento fetal ${ }^{a}$} \\
\hline $\operatorname{Sim}$ & 403 & $6(1,5)$ & $5(1,2)$ & \multirow[t]{2}{*}{0,380} \\
\hline Não & & $162(40,2)$ & $230(57,1)$ & \\
\hline \multicolumn{5}{|c|}{ Percentil de pes $0^{\mathrm{a}}$} \\
\hline$\leq 10$ & 403 & $13(3,2)$ & $14(3,5)$ & \multirow[t]{2}{*}{0,481} \\
\hline$>10$ & & $155(38,5)$ & $221(54,8)$ & \\
\hline \multicolumn{5}{|c|}{ Tipo de parto ${ }^{\mathrm{a}}$} \\
\hline Eutócico & 403 & $97(24,1)$ & $142(35,2)$ & \multirow[t]{2}{*}{0,588} \\
\hline Distócico & & $71(17,6)$ & $93(23,1)$ & \\
\hline \multicolumn{5}{|c|}{ Apgar ao $1^{\circ}$ minut $^{\mathrm{a}}$} \\
\hline$\leq 7$ & 403 & $9(2,2)$ & $5(1,2)$ & \multirow[t]{2}{*}{0,081} \\
\hline$>7$ & & $159(39,5)$ & $230(57,1)$ & \\
\hline \multicolumn{5}{|c|}{ Apgar a $05^{\circ}$ minuto $0^{\mathrm{a}}$} \\
\hline$\leq 7$ & 403 & $1(0,2)$ & $0(0)$ & \multirow[t]{2}{*}{0,236} \\
\hline$>7$ & & $167(58,3)$ & $235(58,3)$ & \\
\hline \multicolumn{5}{|c|}{ Necessidade de cuidados intensivos neonatais ${ }^{a}$} \\
\hline Sim & 403 & $3(0,7)$ & $5(1,2)$ & \multirow[t]{2}{*}{0,303} \\
\hline Não & & $165(40,9)$ & $230(57,1)$ & \\
\hline
\end{tabular}

Frequência absoluta e frequência relativa (\%)

'Teste de Qui-Quadrado

Significância estatística, $p<0,05$

$p=$ nível de significância.

\section{DISCUSSÃO}

A prevalência de sintomatologia depressiva pré-natal, na amostra em estudo, é de aproximadamente $42 \%$, utilizando a PDSS 24. Essa prevalência é superior à reportada para a população portuguesa (entre 14,2\% e 17,9\%)22. A literatura internacional reporta prevalência aproximada de $25 \%{ }^{2}$, com aumento ligeiro no terceiro trimestre de gestação $0^{23-24}$. Estudos recentes referem prevalência de sintomatologia depressiva pré-natal de $23,4 \%{ }^{25}$ no termo da gestação (entre 37 e 39 semanas), valores inferiores à prevalência obtida neste estudo.

Diferentes estudos apontam para o aumento de alterações do sono durante a gestação ${ }^{26}$. Com a progressão da gravidez, registra-se um aumento do tempo acordado e uma diminuição da qualidade do sono ${ }^{27}$, sendo essas alterações mais evidentes nas últimas semanas antes do parto 28,29 . Há também uma diminuição da duração do sono nas fases 3 e 4 e do tempo de sono REM" ${ }^{27,30,31}$, sendo mais notória entre as 35 e 38 semanas comparativamente a 30 e 34 semanas $^{27}$. 
Algumas perturbações do sono, como sleep-disordered breathing, síndrome das pernas inquietas e insônias, também registram um agravamento no termo da gravidez ${ }^{7,26}$. Todas essas alterações encontram-se associadas a um aumento da prevalência de sintomas depressivos nesse período, segundo a literatura7. No presente estudo, a avaliação da consistência interna da PDSS 24 demonstrou que a confiabilidade aumentava com a exclusão do item F4 - "Dificuldades em dormir". Isso revela uma associação plausível entre a prevalência da sintomatologia depressiva pré-natal obtida (superior à da literatura internacional e naciona|2,22-25) e a elevada prevalência de alterações do padrão de sono no período final da gravidez. Essa associação traduz-se, possivelmente, numa sobrestimação da prevalência de sintomatologia depressiva pré-natal, neste estudo.

Foram encontradas associações relevantes entre sintomatologia depressiva no termo da gestação e características sociodemográficas, nomeadamente a escolaridade e o estado civil. Níveis de escolaridade inferiores e grávidas cujo estado civil difira do casado possuem um risco cerca de duas vezes superior de apresentarem sintomatologia depressiva pré-natal. Por outro lado, grávidas que consideram não ter recebido o apoio que desejavam durante a gestação apresentam um risco cerca de três vezes superior de sintomatologia depressiva pré-natal. Dados semelhantes foram encontrados em estudos anteriores ${ }^{23,32-36}$ e reforçam a influência dos fatores sociodemográficos e do apoio social na estabilidade psicológica, capacidade adaptativa e no estabelecimento de um equilíbrio mais salutar entre a gravidez/maternidade e os restante domínios da vida.

Mulheres que não planejaram a atual gestação apresentam maior prevalência de sintomatologia depressiva. Uma gravidez não planejada aumenta o risco de a grávida apresentar sintomatologia depressiva em cerca de duas vezes, o que pode ser explicado pela ausência de preparação psicológica, física e financeira potencialmente associada a uma gestação não planejada. Esses achados suportam a necessidade de planejar a gestação como forma de diminuir o risco de sintomatologia depressiva e suas consequências associadas.

História prévia de depressão revelou ser um preditor independente de sintomatologia depressiva pré-natal, aumentando esse risco em cerca de três vezes. Essa predisposição pode relacionar-se com uma maior vulnerabilidade psicológica dessas mulheres ou pelo fato de o seu contexto psicossocial as tornar vulneráveis a perturbações depressivas. Aliada a essa vulnerabilidade, é sabido que as alterações hormonais fisiológicas da gravidez têm um papel ativo nesse contexto. Esses achados são consistentes com muitos outros estudos ${ }^{37,38}$.

A literatura aponta para que a depressão pré-natal se associa a piores resultados maternos, obstétricos e neonatais, nomeadamente, risco aumentado de pré-eclâmpsia, parto pré-termo, baixo peso ao nascimento, Apgar ao $1^{\circ}$ e $5^{\circ} \mathrm{mi}-$ nuto inferior a 7 e ainda necessidade de internamento em cuidados intensivos neonatais ${ }^{39-45}$. Não se encontrou associação entre essas variáveis e a presença de sintomatologia depressiva, podendo esse achado ser justificado pela alta prevalência de sintomatologia depressiva na amostra.

Este estudo alerta-nos ainda para o cuidado que os profissionais de saúde devem ter aquando do rastreio da sintomatologia depressiva, particularmente no termo da gestação. Ainda que não questionemos a aplicabilidade da PSDSS 24, uma vez que é considerada uma escala adequada para rastrear sintomatologia depressiva pré-nata|21,46, caberá aos médicos avaliarem as grávidas com sintomatologia depressiva por meio de uma entrevista clínica estruturada, de forma a realizarem o diagnóstico de depressão pré-natal. Nessa entrevista diagnóstica, deve ser dada particular atenção às queixas relativas ao sono, que, no termo da gestação, são muito prevalentes. Por outro lado, consideramos que o rastreio de sintomatologia depressiva deve ser realizado numa fase mais precoce da gestação ${ }^{47}$, uma vez que o correto diagnóstico da depressão pré-natal conduz à redução dos sintomas depressivos e a importantes benefícios materno-fetais ${ }^{20}$. Apesar de o rastreio da sintomatologia depressiva pré-natal e posterior diagnóstico de depressão pré-natal ser fundamental, ele é insuficiente por si só para melhorar os desfechos obstétricos e materno-fetais. Os profissionais de saúde devem prestar seguimento apropriado e realizar intervenção precoce, instituindo terapêutica médica quando necessário, bem como referenciar as grávidas para psiquiatria, para abordagens especializadas de terapia cognitivo-comportamental ou ambas, se oportuno ${ }^{48}$.

A prevalência de sintomatologia depressiva pré-natal e as suas ramificações inquietantes impõem uma resposta sistemática por parte da comunidade médica. As grávidas necessitam de ter à sua disposição oportunidades e recursos para se tornarem mais conscientes da sua saúde mental e devem ser estimuladas a implementar estratégias ativas de coping e de adaptação à realidade da gravidez e maternidade, baseadas no autoconhecimento e no contexto pessoal. Tanto os programas de intervenção como os serviços específicos de assistência médica devem ser desenhados à medida das necessidades dessas grávidas. É necessária uma política de remoção de barreiras ao rastreio de sintomatologia depressiva e diagnóstico de depressão pré-natal, por meio da desmistificação da problemática, quer para a grávida, quer para o profissional de saúde. Terá de ser contrariada a visão irreal da gravidez como um período de "completo estado de graça", dando lugar à reflexão e intervenção. De fato, deve ser alterado o paradigma de depressão "pós-natal" para depressão "perinatal", pois a depressão pré-natal existe e acarreta repercussões na saúde materna e fetal, bem como na dinâmica familiar ${ }^{11-15}$.

Este estudo apresenta vantagens e limitações. Apresenta como pontos fortes o tamanho amostral e a sua natureza prospectiva. É igualmente importante referir que este estudo, 
segundo a nossa pesquisa, é o primeiro a proceder ao rastreio de sintomatologia depressiva pré-natal em grávidas de baixo risco, a termo da gestação, numa amostra portuguesa.

Uma das limitações deste estudo centra-se na associação plausível entre sintomatologia depressiva pré-natal e as alterações do padrão de sono no termo da gestação, traduzida pelo aumento da confiabilidade com a exclusão do item F4 - "Dificuldades em dormir", da PDSS 24. Adicionalmente, sendo a PDSS 24 um instrumento de rastreio, os resultados estão sujeitos a erros. Se, por um lado, os resultados falso-negativos conduzem a uma subestimação da prevalência de sintomatologia depressiva pré-natal, os falso-positivos aumentam erroneamente as estimativas. Além disso, visto que os instrumentos de rastreio visam identificar indivíduos, em uma população, com risco aumentado para determinada doença, a sua eficácia depende da prevalência da doença na população considerada. Ora, segundo a literatura, a prevalência de sintomatologia depressiva pré-natal ronda os $25 \%{ }^{2}$, conforme discutido anteriormente. Assim, quanto menor a prevalência populacional, mais baixo se torna o valor preditivo positivo e mais elevada a contagem de falso-positivos. De fato, o valor de falso-positivos (20,8\%), associado à utilização da PDSS 24 no rastreio de sintomatologia depressiva pré-natal, é elevado. No entanto, a literatura aponta para que grávidas com falso-positivos apresentem níveis mais elevados de psicopatologia e distress, o que justifica a sua identificação e orientação ${ }^{21}$. Por outro lado, embora a identificação dos falso-positivos esteja associada a consumo superior de recursos e potencialmente acarrete mais distress para algumas grávidas, acreditamos que essas consequências serão menos nefastas do que a não realização de rastreio de sintomatologia depressiva no período pré-natal e a não identificação posterior de grávidas com depressão.

É também importante aludir à impossibilidade de exclusão de vieses associados à autoaplicação dos questionários, em detrimento de avaliações clínicas objetivas. Adicionalmente, a realização de entrevista clínica estruturada para diagnóstico de depressão pré-natal nas grávidas com sintomatologia depressiva seria, sem dúvida, uma mais valia para este estudo. Essa limitação abre portas para a realização de novas investigações, que visem à determinação da prevalência de depressão pré-natal (com base em diferentes sistemas de diagnóstico, incluindo (ID-11 e DSM-5), após aplicação de metodologias de rastreio.

Assim sendo, tendo em conta as limitações evidenciadas em relação à utilização da PDSS 24 no termo da gravidez, os nossos resultados, apesar de inovadores, devem ser interpretados com comedimento.

\section{CONCLUSÃO}

O rastreio da sintomatologia depressiva deve ser conduzido durante a gravidez, dada a elevada prevalência e suas con- sequências negativas da depressão pré-natal em nível obstétrico e materno-fetal. Porém, no termo da gestação, o uso da PDSS 24 como ferramenta de rastreio de sintomatologia depressiva deve ser feito com cautela. A elevada prevalência de sintomas relacionados com o sono (por exemplo, qualidade do sono) nessa fase da gestação pode conduzir à sobrestimação da prevalência da sintomatologia depressiva, usando a PDSS 24. Importa, por isso, acompanhar esse rastreio de uma avaliação clínica cuidada.

\section{CONTRIBUIÇÕES INDIVIDUAIS}

Vanessa Silva e Cátia Ferreira - Participaram no desenho do estudo, recolha, análise e interpretação dos dados, redação do artigo e revisão crítica do conteúdo intelectual.

Adriana Basílio e Ana Beatriz Ferreira - Participaram na análise e interpretação de dados e redação do artigo.

Berta Maia e Rui Miguelote - Participaram no desenho do estudo, orientação e revisão crítica do conteúdo intelectual.

Todos os autores leram e aprovaram a versão final do manuscrito a ser publicada.

\section{CONFLITO DE INTERESSES}

Os autores declaram não existir conflito de interesses.

\section{REFERÊNCIAS}

1. Wisner KL, Peindl KS, Gigliotti T, Hanusa BH. Obsessions and compulsions in women with postpartum depression. J Clin Psychiatry. 1999;60(3):176-80.

2. Gaynes BN, Gavin N, Meltzer-Brody S, Lohr KN, Swinson T, Gartlehner G, et al. Perinatal depression: prevalence, screening accuracy, and screening outcomes. Evid Rep Technol Assess (Summ). 2005(119):1-8.

3. Georgiopoulos AM, Bryan TL, Wollan P, Yawn BP. Routine screening for postpartum depression. J Fam Pract. 2001;50(2):117-22.

4. Pereira AT, Marques M, Soares MJ, Maia BR, Bos S, Valente J, et al. Profile of depressive symptoms in women in the perinatal and outside the perinatal period: similar or not? J Affect Disord. 2014;166:71-8.

5. Lee DTSC, Chung TKH. Screening for perinatal depression. In: Henshaw C, Elliot S, editors. Screening in developing countries London: Jessica Kingsley Publishers; 2005. p. 90-8.

6. Goodman JH. Women's attitudes, preferences, and perceived barriers to treatment for perinatal depression. Birth. 2009;36(1):60-9.

7. Marques M, Bos S, Soares MJ, Maia B, Pereira AT, Valente J, et al. Is insomnia in late pregnancy a risk factor for postpartum depression/depressive symptomatology? Psychiatry Res. 2011;186(2-3):272-80.

8. Faisal-Cury A, Menezes PR. Antenatal depression strongly predicts postnatal depression in primary health care. Rev Bras Psiquiatr. 2012;34(4):446-50.

9. Wisner KL, Chambers C, Sit DK. Postpartum depression: a major public health problem. JAMA. 2006;296(21):2616-8.

10. Bernstein IH, Rush AJ, Yonkers K, Carmody TJ, Woo A, McConnell K, et al. Symptom features of postpartum depression: are they distinct? Depress Anxiety. 2008;25(1):20-6. 
11. Andersson L, Sundstrom-Poromaa I, Wulff M, Astrom M, Bixo M. Implications of antenatal depression and anxiety for obstetric outcome. Obstet Gynecol. 2004;104(3):467-76.

12. Pereira AT, Soares MJ, Bos S, Maia BR, Valente J, Nogueira V, et al. Why should we screen for perinatal depression? Ten reasons to do it. Int J Clin Neurosci Ment Health. 2014.

13. Eastwood J, Ogbo FA, Hendry A, Noble J, Page A. The Impact of Antenatal Depression on Perinatal Outcomes in Australian Women. PloS One. 2017;12(1):e0169907.

14. Glover V, Bergman K, O'Connor TG. The Effects of Maternal Stress, Anxiety, and Depression During Pregnancy on the Neurodevelopment of the Child. In: Stone SD, Menken AE, eds. Perinatal and Postpartum Mood Disorders: Perspectives and Treatment Guide for the Health Care Practitioner. New York: Springer; 2008.

15. Burke $L$. The impact of maternal depression on familial relationships. Int Rev Psychiatry. 2003;15(3):243-55.

16. Beck CT GR. Postpartum Depression Screening Scale manual. In: Services WP, editor. Los Angeles; 2002

17. Pereira AT, Bos SC, Marques M, Maia BR, Soares MJ, Valente J, et al. The postpartum depression screening scale: is it valid to screen for antenatal depression? Arch Womens Ment Health. 2011;14(3):227-38.

18. Gjerdingen DK, Yawn BP. Postpartum depression screening: importance, methods, barriers, and recommendations for practice. J Am Board Fam Med. 2007;20(3):280-8.

19. Dennis $\mathrm{CL}$, Hodnett E. Psychosocial and psychological interventions for treating postpartum depression. Cochrane Database Syst Rev. 2007(4):CD006116.

20. Buist AE, Barnett BE, Milgrom J, Pope S, Condon JT, Ellwood DA, et al. To screen or not to screen - that is the question in perinatal depression. Med J Aust. 2002;177 Suppl:S101-5.

21. Pereira AT, Bos S, Marques M, Maia B, Soares MJ, Valente J, et al. Short forms of the postpartum depression screening scale: as accurate as the original form. Arch Womens Ment Health. 2013;16(1):67-77.

22. Maia BR, Marques M, Bos S, Pereira AT, Soares MJ, Valente J, et al. Epidemiology of perinatal depression in Portugal: categorical and dimensional approach. Acta Med Port. 2011;24 Suppl 2:443-8.

23. Gluckman PD, Hanson MA, Cooper C, Thornburg KL. Effect of in utero and early-life conditions on adult health and disease. N Engl J Med. 2008;359(1):61-73.

24. Ryan D, Milis L, Misri N. Depression during pregnancy. Can Fam Phys. 2005;51(8):1087-93.

25. Miksic S, Miskulin M, Juranic B, Rakosec Z, Vcev A, Degmecic D. Depression and Suicidality during Pregnancy. Psychiatria Danubina. 2018;30(1):85-90.

26. Izci Balserak B, Kathryn L. Sleep Disturbances and sleep-related disorders in pregnancy. Kryger M, Roth T, Dement W. Principles and practice of sleep medicine. 5th ed. Toronto: Elsevier; 2010.

27. Hertz G, Fast A, Feinsilver SH, Albertario CL, Schulman H, Fein AM. Sleep in normal late pregnancy. Sleep. 1992;15(3):246-51.

28. Evans ML, Dick MJ, Clark AS. Sleep during the week before labor: relationships to labor outcomes. Clin Nurs Res. 1995;4(3):238-49; discussion 50-2.

29. Mindell JA, Jacobson BJ. Sleep disturbances during pregnancy. J Obstet Gynecol Neonatal Nurs. 2000;29(6):590-7.

30. Brunner DP, Munch M, Biedermann K, Huch R, Huch A, Borbely AA. Changes in sleep and sleep electroencephalogram during pregnancy. Sleep. 1994;17(7):576-82.
31. Driver HS, Shapiro CM. A longitudinal study of sleep stages in young women during pregnancy and postpartum. Sleep. 1992;15(5):449-53.

32. Austin MP, Lumley J. Antenatal screening for postnatal depression: a systematic review. Acta Psychiatr Scand. 2003;107(1):10-7.

33. Cooper PJ, Murray L, Hooper R, West A. The development and validation of a predictive index for postpartum depression. Psychol Med. 1996;26(3):627-34.

34. Halbreich $U$. The association between pregnancy processes, preterm delivery, low birth weight, and postpartum depressions - the need for interdisciplinary integration. Am J Obstet Gynecol. 2005;193(4):1312-22.

35. Reid AJ, Biringer A, Carroll JD, Midmer D, Wilson LM, Chalmers B, et al. Using the ALPHA form in practice to assess antenatal psychosocial health. Antenatal Psychosocial Health Assessment. CMAJ. 1998;159(6):677-84

36. O'hara MW, Swain AM. Rates and Risk of postpartum depression - a meta-analysis. Int Rev Psychiatry. 2009;8:37-54

37. Johanson R, Chapman G, Murray D, Johnson I, Cox J. The North Staffordshire Maternity Hospital prospective study of pregnancy-associated depression. J Psychosom Obstet Gynaecol. 2000;21(2):93-7.

38. Dayan J, Creveuil C, Dreyfus M, Herlicoviez M, Baleyte JM, O'Keane V. Developmenta model of depression applied to prenatal depression: role of present and past life events, past emotional disorders and pregnancy stress. PloS One. 2010;5(9):e12942.

39. Kurki T, Hiilesmaa V, Raitasalo R, Mattila $H$, Ylikorkala 0 . Depression and anxiety in early pregnancy and risk for preeclampsia. Obstet Gynecol. 2000;95(4):487-90.

40. Gaffney KF, Kitsantas P, Brito A, Swamidoss CS. Postpartum depression, infant feeding practices, and infant weight gain at six months of age. J Pediatr Health Care. 2014;28(1):43-50

41. Dias CC, Fiqueiredo B. Breastfeeding and depression: a systematic review of the literature. J Affect Disord. 2015;171:142-54.

42. Adewuya A0, 0 la BO, Aloba 00, Mapayi BM, Okeniyi JA. Impact of postnatal depression on infants' growth in Nigeria. J Affect Disord. 2008;108(1-2):191-3.

43. Rahman A, labal Z, Bunn J, Lovel H, Harrington R. Impact of maternal depression on infant nutritional status and illness: a cohort study. Arch Gen Psychiatry. 2004;61(9):946-52.

44. Zubaran C, Foresti K. The correlation between breastfeeding self-efficacy and maternal postpartum depression in southern Brazil. Sex Reprod Healthc. 2013;4(1):9-15.

45. Grigoriadis S, VonderPorten EH, Mamisashvili L, Tomlinson G, Dennis CL, Koren G, et al. The impact of maternal depression during pregnancy on perinatal outcomes: a systematic review and meta-analysis. J Clin Psychiatry. 2013;74(4):e321-41.

46. Azevedo J, Xavier S, Marques C, Bento E, Marques M, Soares MJ, et al. Confirmatory factor analysis of the perinatal depression screening scale-24. Eur Psychiatry. 2017;41(Suppl):361.

47. Hewitt C, Gilbody S, Brealey S, Paulden M, Palmer S, Mann R, et al. Methods to identify postnatal depression in primary care: an integrated evidence synthesis and value of information analysis. Health Technol Assess. 2009;13(36):1-145, 7-230.

48. The American College of Obstetricians and Gynecologists Committee Opinion no. 630. Screening for perinatal depression. Obstet Gynecol. 2015;125(5):1268-71. 